Annals of Pure and Applied Mathematics

Vol. 15, No. 2, 2017, 305-314

ISSN: 2279-087X (P), 2279-0888(online)

Published on 11 December 2017

www.researchmathsci.org

DOI: http://dx.doi.org/10.22457/apam.v15n2a17

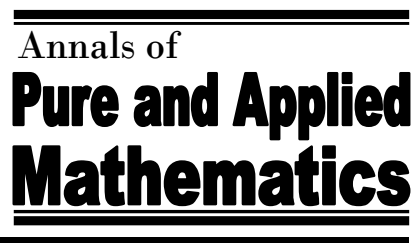

\title{
A Purchasing Inventory Model for Breakable items with Permissible Delay in Payments and Price Discount
}

\author{
M.Maragatham $^{1}$ and G.Gnanvel ${ }^{2}$ \\ ${ }^{1}$ Department of Mathematics, Periyar E.V.R.College \\ Trichy-23, Tamilnadu, India. \\ ${ }^{2}$ Department of Mathematics, SRG Engg College, Namakkal-17 \\ Tamilnadu, India.
}

Received 25 November 2017; accepted 10 December 2017

\begin{abstract}
In this paper, a purchasing inventory model with an aim to minimize the total inventory cost and to find the optimal time interval is discussed. Here the retailers are given trade credit offer. In this model, supplier provides replacement, or price discount for damageable items. Shortages are allowed and backlogged. The results are illustrated with numerical example.
\end{abstract}

Keywords: inventory control, permissible delay in payments, deteriorating items, trade credit period, price discount.

\section{AMS Mathematics Subject Classification (2010): 90B05}

\section{Introduction}

An important assumption in inventory models found in the existing literature is that the life time of an item is infinite while it is in storage. But the effect of deterioration plays an important role in the storage of some commonly used decaying items like, breakable items (glass, china clay, ceramic goods...etc). Due to the deterioration in stored products some of these items loses their quality and cannot complete the customer's need for ideal product. Deterioration in these items may be constant, continuous, time dependent or stock dependent. The economic order quantity model is based on the assumption that the retailer paid for the items immediately after the items are received. However, in practice, the supplier may provide the retailer many incentives such as a cash discount to motivate faster payment and stimulate sales, or a permissible delay in payment to attract new customer and increase the sales. Hence trade credit can play a major role in inventory control for both the supplier as well as retailer. [1] Goyal first developed the EOQ model under the conditions of permissible delay in payments, [2] Ray (2014) considered a nonlinear EOQ model with effect of trade credit. In [3] Sajadifar, Ahmadavaji developed an inventory model with demand dependent replenishment rate for damageable items and shortages. In [4] Mukesh Kumar et al developed a deterministic inventory model for deteriorating items with price dependent demand and time varying holding cost under trade credit. In [6] Tangam A considered Retailers inventory system in a two level trade credit financing with selling price discount and partial order cancellations. [5] Palanivel $\mathrm{M}$ et al consider an EOQ model for non-instantaneous deteriorating items with power 


\section{M.Maragatham and G.Gnanvel}

demand time dependent holding cost, partial backlogging and permissible delay in payments. Recently many authors developed an EOQ model for deteriorating items with quadratic demand rate under permissible delay in payments.

In this modelcustomer may pay cashwhen the supplier offered trade credit to the retailer. The shortages are allowed when unsatisfied demand will be backlogged in each cycle. The demand rate is time dependent, and the suitable numerical example is given to illustrate the model.

\section{Notations and assumptions}

\subsection{Notations}

The following notations have been used in developing the model.

A - The ordering cost per order.

$\mathrm{P}-$ The purchasing rate per unit time per year.

Q- The initial inventory level.

$D(t)$-The demand rate at any time $t \geq 0, D(t)=a+b t, a, b>0$ where $a, b$ are positive constants.

$\mathrm{T}$ - The length of replenishment cycle.

r- The price discount.

h- The holding cost per unit per unit time.

$\theta$ - The rate of damageable items.

$\mathrm{I}_{\mathrm{e}^{-}}$Interest which can be earned per year.

$\mathrm{I}_{\mathrm{p}^{-}}$Interest payable per year.

$\mathrm{C}_{1}$ - The shortage cost for backlogged items per unit per year.

$\mathrm{t}_{1^{-}}$- The time at which the inventory level reaches zero.

$\mathrm{t}_{\mathrm{r}}-$ The replacement time and trade credit period.

$\mathrm{m}_{\mathrm{o}}$-The mark up of selling price for damaged items.

$B(Q)-T h e$ number of damaged units per unit of time at time $t$ and is a function of current inventory level Q

$\mathrm{TC}$ - The minimum total cost per unit time.

\subsection{Assumptions}

The following assumptions are made in developing the model

1. Thesupplier offers price discount to his retailer at $\mathrm{t}_{1}<\mathrm{t}_{\mathrm{r}}$.

2. The breakable items are replaced when end of the trade credit period.

3. The time horizon is infinite.

4. The lead time is zero.

5. Shortages are allowed and backlogged.

6. Selling price for damaged items $S_{d}$ is a multiple of purchasing cost. $S_{d}=m_{0} * P$ $0 \leq \mathrm{m}_{0} \leq 1$.

\section{Mathematical formulation}

Based upon the above notations and assumptions are to be defined as follows.

Case I

$$
t_{1}>t_{r}
$$

The retailer replaced breakable items at end of the permissible delay period.

Case II

$$
\mathrm{t}_{1}<\mathrm{t}_{\mathrm{r}}
$$


A Purchasing Inventory Model for Breakable items with Permissible Delay in Payments and Price Discount

The retailer adopts each discount to settle the accounts and the payments in time $t_{r}$

Case III

$$
\mathrm{t}_{1}=\mathrm{t}_{\mathrm{r}}
$$

The retailer replacesbreakable items at the end of the permissible period, at the time of inventory level is zero.

3.1. Case I $t_{1}>t_{r}$

In this section, the detailed mathematical formulation for the inventory problem is given. The inventory level is dropping to zero because of demand and damageable items during the interval $\left[0, t_{1}\right]$. The period $\left[0, t_{r}\right]$ is delay in payments and $t_{r}$ is the replacement time for damageable items. Finally the shortage occurs due to demand and backlogging during the time interval $\left[\mathrm{t}_{1}, \mathrm{~T}\right]$, based on the above description, the inventory level at time $\mathrm{t}, \mathrm{I}(\mathrm{t})$ will be described by the following differential equation.

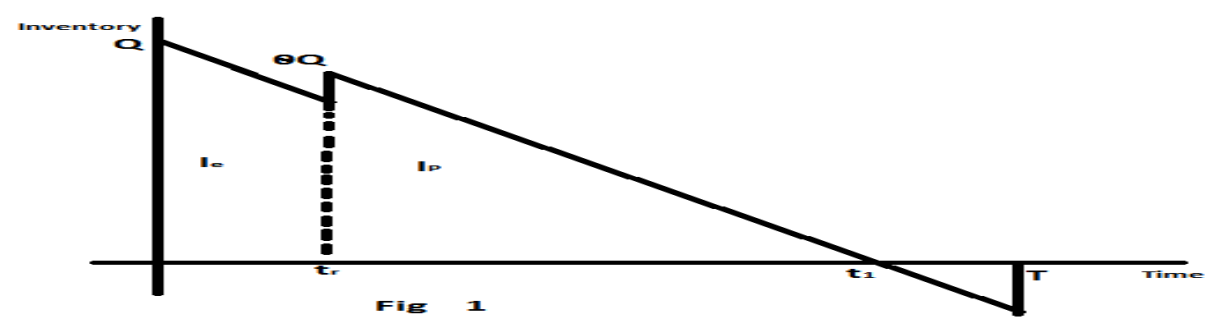

Figure 1:

$\frac{\mathrm{d}(\mathrm{t})}{\mathrm{dt}}+\theta \mathrm{I}(\mathrm{t})=-\mathrm{D}(\mathrm{t}) \quad 0<t<\mathrm{t}_{\mathrm{r}}$

With the condition $\mathrm{t}=0$ and $\mathrm{I}(\mathrm{t})=\mathrm{Q}$ the solution of $(1)$ is

$$
\mathrm{I}(\mathrm{t})=\mathrm{Q}(1-\theta \mathrm{t})-\text { at } \quad 0<t<\mathrm{t}_{\mathrm{r}}
$$

During the time interval $\left[t_{r}, t_{1}\right]$, the differential equation representing the inventory status

is given by $\frac{\mathrm{d}(\mathrm{t})}{\mathrm{dt}}+\theta \mathrm{I}(\mathrm{t})=-\mathrm{D}(\mathrm{t}) \quad \mathrm{t}_{\mathrm{r}}<t<\mathrm{t}_{1}$

With the boundary condition $\mathrm{I}\left(\mathrm{t}_{\mathrm{r}}\right)=\mathrm{Q}(1-\theta \mathrm{t})-$ at $+\mathrm{Q} \theta$ the solution of (2) is

$I(t)=\left[Q\left(1-\theta t_{r}+\theta\right)-a t_{r}+\frac{b t_{r}}{\theta}\right]\left(\theta t_{r}-\theta t+1\right)-\frac{b t_{r}}{\theta}+a\left(t_{r}-t\right) t_{r}<t<t_{1}$

With the condition $\mathrm{t}=\mathrm{t}_{1}$ and $\mathrm{I}\left(\mathrm{t}_{1}\right)=0$ the solution

$\mathrm{t}_{1}=\frac{\left[\mathrm{Q}\left(1-\theta \mathrm{t}_{\mathrm{r}}+\theta\right)-\mathrm{at} \mathrm{t}_{\mathrm{r}}+\frac{\mathrm{btr}}{\theta}\right]\left(\theta \mathrm{t}_{\mathrm{r}}+1\right)-\left(\frac{\mathrm{b}}{\theta}-\mathrm{a}\right) \mathrm{t}_{\mathrm{r}}}{\left[\mathrm{Q}\left(1-\theta \mathrm{t}_{\mathrm{r}}+\theta\right)-\mathrm{at} \mathrm{t}_{\mathrm{r}}+\frac{\mathrm{bt} \mathrm{t}_{\mathrm{r}}}{\theta}+\mathrm{a}\right]}$

During the third interval $\left[\mathrm{t}_{1}, \mathrm{~T}\right]$, shortages occurred and the demand is backlogged, when $\mathrm{t}=\mathrm{T}$. The inventory level at time $\mathrm{t}$ is governed by the following differential equation

$\frac{\mathrm{d}(\mathrm{t})}{\mathrm{dt}}=-\mathrm{D}(\mathrm{t}) \mathrm{t}_{1}<t<T$

With the boundary conditions are $I\left(t_{1}\right)=0, I(T)=-(1-\theta)\left[a+b\left(T-t_{1}\right)\right]$

$$
\mathrm{I}(\mathrm{t})=\left[\mathrm{a}(\mathrm{T}-\mathrm{t})+\frac{\mathrm{b}}{2}\left(\mathrm{~T}^{2}-\mathrm{t}^{2}\right)-(1-\theta)\left[\mathrm{a}+\mathrm{b}\left(\mathrm{T}-\mathrm{t}_{1}\right)\right] \mathrm{t}_{1}<t<T\right.
$$

The ordering cost is $\mathrm{OC}=\mathrm{A}$

The purchasing cost is $\mathrm{PC}=\mathrm{P} * \mathrm{Q}+(1+\theta)\left[\mathrm{a}+\mathrm{b}\left(\mathrm{T}-\mathrm{t}_{1}\right)\right]$

The total inventory holding cost for the cycle $\left[0, t_{1}\right]$ is $H C=h \int_{0}^{t_{1}} I(t) d t$ 
M.Maragatham and G.Gnanvel

$$
H C=h\left\{\int_{0}^{t_{r}} I(t) d t+\int_{t_{r}}^{t_{1}} I(t) d t\right\}
$$

$$
\begin{aligned}
& H C=h\{ Q\left(t_{r}-\frac{\theta t_{r}^{2}}{2}\right)-\frac{a t_{r}^{2}}{2}+\left(Q\left(1-\theta t_{r}+\theta\right)-a t_{r}+\frac{b t_{r}}{\theta}\right)\left(\frac{\theta}{2}\left(t_{1}-t_{r}\right)^{2}-\right. \\
&\left.\left.\theta t_{r}\left(t_{r}-2\right)+2 t_{1}\right)-\left(t_{1}-t_{r}\right)\left(\frac{b t_{r}}{\theta}+\frac{a}{2}\left(t_{1}-t_{r}\right)\right)\right\}
\end{aligned}
$$

The total shortage cost SC during the period $\left[\mathrm{t}_{1}, \mathrm{~T}\right]$ is given bySC $=\mathrm{C}_{1} \int_{\mathrm{t}_{1}}^{\mathrm{T}} \mathrm{I}(\mathrm{t}) \mathrm{dt}$

$\mathrm{SC}=\mathrm{C}_{1}\left[\frac{\mathrm{b}}{6}\left(2 \mathrm{~T}^{3}-3 \mathrm{~T}^{2} \mathrm{t}_{1}+\mathrm{t}_{1}{ }^{3}\right)+\left(\frac{\mathrm{a}}{2}-\mathrm{b}(1-\theta)\right)\left(\mathrm{T}-\mathrm{t}_{1}\right)^{2}-\mathrm{a}(1-\theta)\left(\mathrm{T}-\mathrm{t}_{1}\right)\right]$

The interest earned per unit time in $\left[0, t_{r}\right]$ isIE $=\mathrm{SI}_{\mathrm{e}} \int_{0}^{\mathrm{t}_{\mathrm{r}}} \mathrm{D}(\mathrm{t}) \mathrm{dt}$

$\mathrm{IE}=\mathrm{SI}_{\mathrm{e}}\left[\mathrm{at} \mathrm{t}_{\mathrm{r}}+\frac{\mathrm{bt}_{\mathrm{r}}{ }^{2}}{2}\right]$

The interest payable per cycle per unit time for the inventory not being after due date say $\mathrm{t}_{\mathrm{r}}$ isIP $=\mathrm{PI}_{\mathrm{p}} \int_{\mathrm{t}_{\mathrm{r}}}^{\mathrm{t}_{1}} \mathrm{I}(\mathrm{t}) \mathrm{dt}$

$$
\begin{aligned}
I P=P I_{p} & {\left[\left(Q\left(1-\theta t_{r}+\theta\right)-a t_{r}+\frac{b t_{r}}{\theta}\right)\left(\frac{\theta}{2}\left(t_{1}-t_{r}\right)^{2}-\theta t_{r}\left(t_{r}-2\right)+2 t_{1}\right)-\right.} \\
& \left.\left(t_{1}-t_{r}\right)\left(\frac{b t_{r}}{\theta}+\frac{a}{2}\left(t_{1}-t_{r}\right)\right)\right]
\end{aligned}
$$

So, the total variable cost per unit time is $\mathrm{TC}=\frac{1}{\mathrm{~T}}[\mathrm{OC}+\mathrm{PC}+\mathrm{HC}+\mathrm{SC}+\mathrm{IP}-\mathrm{IE}] \mathrm{TC}=$

$$
\begin{aligned}
& \frac{1}{\mathrm{~T}}\left\{\mathrm{~A}+\mathrm{P} * \mathrm{Q}+(1-\theta)\left[\mathrm{a}+\mathrm{b}\left(\mathrm{T}-\mathrm{t}_{1}\right)\right]+\mathrm{h}\left\{\mathrm{Q}\left(\mathrm{t}_{\mathrm{r}}-\frac{\theta \mathrm{t}_{\mathrm{r}}{ }^{2}}{2}\right)-\frac{\mathrm{at}_{\mathrm{r}}{ }^{2}}{2}+\left(\mathrm { Q } \left(1-\theta \mathrm{t}_{\mathrm{r}}+\right.\right.\right.\right. \\
&\left.\quad \theta)-\mathrm{at}_{\mathrm{r}}+\frac{\mathrm{bt} \mathrm{t}_{\mathrm{r}}}{\theta}\right)\left(\frac{\theta}{2}\left(\mathrm{t}_{1}-\mathrm{t}_{\mathrm{r}}\right)^{2}-\theta \mathrm{t}_{\mathrm{r}}\left(\mathrm{t}_{\mathrm{r}}-2\right)+2 \mathrm{t}_{1}\right)-\left(\mathrm{t}_{1}-\mathrm{t}_{\mathrm{r}}\right)\left(\frac{\mathrm{bt}_{\mathrm{r}}}{\theta}+\right. \\
&\left.\left.\frac{\mathrm{a}}{2}\left(\mathrm{t}_{1}-\mathrm{t}_{\mathrm{r}}\right)\right)\right\}+\mathrm{C}_{1}\left[\frac{\mathrm{b}}{6}\left(2 \mathrm{~T}^{3}-3 \mathrm{~T}^{2} \mathrm{t}_{1}+\mathrm{t}_{1}{ }^{3}\right)+\left(\frac{\mathrm{a}}{2}-\mathrm{b}(1-\theta)\right)\left(\mathrm{T}-\mathrm{t}_{1}\right)^{2}-\right. \\
&\left.\mathrm{a}(1-\theta)\left(\mathrm{T}-\mathrm{t}_{1}\right)\right]+\mathrm{PI}_{\mathrm{p}}\left[( \mathrm { Q } ( 1 - \theta \mathrm { t } _ { \mathrm { r } } + \theta ) - \mathrm { at } _ { \mathrm { r } } + \frac { \mathrm { bt } _ { \mathrm { r } } } { \theta } ) \left(\frac{\theta}{2}\left(\mathrm{t}_{1}-\mathrm{t}_{\mathrm{r}}\right)^{2}-\right.\right. \\
&\left.\left.\left.\theta \mathrm{t}_{\mathrm{r}}\left(\mathrm{t}_{\mathrm{r}}-2\right)+2 \mathrm{t}_{1}\right)-\left(\mathrm{t}_{1}-\mathrm{t}_{\mathrm{r}}\right)\left(\frac{\mathrm{bt}}{\theta}+\frac{\mathrm{a}}{2}\left(\mathrm{t}_{1}-\mathrm{t}_{\mathrm{r}}\right)\right)\right]-\mathrm{SI}_{\mathrm{e}}\left[\mathrm{at}_{\mathrm{r}}+\frac{\mathrm{bt}_{\mathrm{r}}{ }^{2}}{2}\right]\right\}
\end{aligned}
$$

For minimizing the total relevant cost per unit time, the approximate optimal values of $\mathrm{T}$ can be obtained by solving the following equation $\frac{d T C}{d T}=0$

$\frac{\mathrm{dTC}}{\mathrm{dT}}=-\frac{1}{\mathrm{~T}^{2}}\left\{\mathrm{~A}+\mathrm{P} * \mathrm{Q}+(1-\theta)\left(\mathrm{a}-\mathrm{bt}_{1}\right)+\mathrm{h}\left[\mathrm{Q}\left(\mathrm{t}_{\mathrm{r}}-\frac{\theta \mathrm{t}_{\mathrm{r}}{ }^{2}}{2}\right)-\frac{\mathrm{at}_{\mathrm{r}}{ }^{2}}{2}+\left(\mathrm{Q}\left(1-\theta \mathrm{t}_{\mathrm{r}}+\right.\right.\right.\right.$ $\left.\left.\theta)-a t_{r}+\frac{b t_{r}}{\theta}\right)\left(\frac{\theta}{2}\left(t_{1}-t_{r}\right)^{2}-\theta t_{r}\left(t_{r}-2\right)+2 t_{1}\right)-\left(t_{1}-t_{r}\right)\left(\frac{b t_{r}}{\theta}+\frac{a}{2}\left(t_{1}-t_{r}\right)\right)\right]+$ $P I_{p}\left[\left(Q\left(1-\theta t_{r}+\theta\right)-a t_{r}+\frac{b t_{r}}{\theta}\right)\left(\frac{\theta}{2}\left(t_{1}-t_{r}\right)^{2}-\theta t_{r}\left(t_{r}-2\right)+2 t_{1}\right)-\left(t_{1}-t_{r}\right)\left(\frac{b t_{r}}{\theta}+\right.\right.$ $\left.\left.\left.\frac{\mathrm{a}}{2}\left(\mathrm{t}_{1}-\mathrm{t}_{\mathrm{r}}\right)\right)\right]-\mathrm{SI}_{\mathrm{e}}\left[\mathrm{at}_{\mathrm{r}}+\frac{\mathrm{bt}_{\mathrm{r}}{ }^{2}}{2}\right]+\mathrm{C}_{1}\left[\frac{\mathrm{b}}{6} \mathrm{t}_{1}{ }^{3}+\left(\frac{\mathrm{a}}{2}-\mathrm{b}(1-\theta)\right) \mathrm{t}_{1}{ }^{2}+\mathrm{a}(1-\theta) \mathrm{t}_{1}\right]\right\}+$ $\mathrm{C}_{1}\left[\frac{\mathrm{a}}{2}-\mathrm{b}\left(1-\theta-\frac{\mathrm{t}_{1}}{2}\right)\right]+\mathrm{C}_{1} 2 \mathrm{bT}=0$

This also satisfies the conditions $\frac{d^{2} T C}{d T^{2}}=\frac{2}{T^{3}}\left\{A+P * Q+(1-\theta)\left(a-b t_{1}\right)+h\left[Q\left(t_{r}-\frac{\theta t_{r}{ }^{2}}{2}\right)-\frac{a t_{r}{ }^{2}}{2}+\left(Q\left(1-\theta t_{r}+\right.\right.\right.\right.$
$\left.\left.\theta)-a t_{r}+\frac{b t_{r}}{\theta}\right)\left(\frac{\theta}{2}\left(t_{1}-t_{r}\right)^{2}-\theta t_{r}\left(t_{r}-2\right)+2 t_{1}\right)-\left(t_{1}-t_{r}\right)\left(\frac{b t_{r}}{\theta}+\frac{a}{2}\left(t_{1}-t_{r}\right)\right)\right]+$
$P I_{p}\left[\left(Q\left(1-\theta t_{r}+\theta\right)-a t_{r}+\frac{b t_{r}}{\theta}\right)\left(\frac{\theta}{2}\left(t_{1}-t_{r}\right)^{2}-\theta t_{r}\left(t_{r}-2\right)+2 t_{1}\right)-\left(t_{1}-\right.\right.$ 
A Purchasing Inventory Model for Breakable items with Permissible Delay in Payments and Price Discount

$\left.\left.t_{r}\right)\left(\frac{b t_{r}}{\theta}+\frac{a}{2}\left(t_{1}-t_{r}\right)\right)\right]-S I_{e}\left[a t_{r}+\frac{b t_{r}{ }^{2}}{2}\right]+C_{1}\left[\frac{b}{6} t_{1}{ }^{3}+\left(\frac{a}{2}-b(1-\theta)\right) t_{1}{ }^{2}+\right.$ $\left.\left.a(1-\theta) t_{1}\right]\right\}+C_{1} 2 b>0$

\subsection{Case II}

$t_{1}<t_{r}$

In this case, the inventory level reduces due to demand rate as well as damageable/breakable items rate during the inventory $\left[0, \mathrm{t}_{1}\right]$. At the period, price discount is allowed for damageable/breakable items. The time $t_{r}$ is permissible delay in payments for the retailer. Finally shortages occur due to demand and backlogging during the time inventory $\left[\mathrm{t}_{1}, \mathrm{~T}\right]$. The differential equation representing the inventory status is

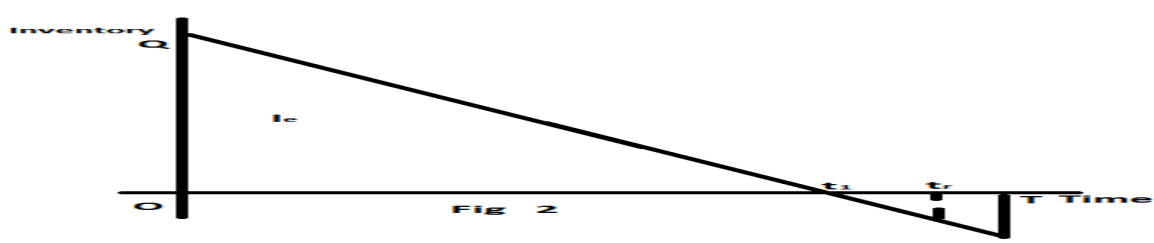

Figure 2:

$\frac{\mathrm{d}(\mathrm{t})}{\mathrm{dt}}+\theta \mathrm{I}(\mathrm{t})=-\mathrm{D}(\mathrm{t}) \quad 0<t<\mathrm{t}_{1}$

With the condition $t=0$ and $I(t)=Q$ the solution of $(1)$ is

$\mathrm{I}(\mathrm{t})=\mathrm{Q}(1-\theta \mathrm{t})-\mathrm{at}$

The boundary conditions are as follows $\quad I\left(t_{1}\right)=0$

$\mathrm{t}_{1}=\frac{\mathrm{Q}}{\theta \mathrm{Q}+\mathrm{a}}$

During the interval $\left[\mathrm{t}_{1}, T\right]$ the backlogged at time $\mathrm{I}(\mathrm{t})$ is governed by the following differential equation From Equation (6)

$I(t)=\left[a\left(t_{1}-t\right)+\frac{b}{2}\left(t_{1}^{2}-t^{2}\right)\right]$

The ordering cost is $\mathrm{OC}=\mathrm{A}$

The purchasing cost is $\mathrm{PC}=\mathrm{P} * \mathrm{Q}$

The holding cost for the period $\left[0, \mathrm{t}_{1}\right]$ is $\mathrm{HC}=\mathrm{h} \int_{0}^{\mathrm{t}_{1}} \mathrm{I}(\mathrm{t}) \mathrm{dt}$

$\mathrm{HC}=\mathrm{h}\left[\mathrm{Q}\left(\mathrm{t}_{1}-\frac{\theta \mathrm{t}_{1}{ }^{2}}{2}\right)-\frac{\theta \mathrm{t}_{1}{ }^{2}}{2}\right]$

The shortage cost for the period $\left[\mathrm{t}_{1}, \mathrm{~T}\right]$ is $\quad \mathrm{SC}=\mathrm{C}_{1} \int_{\mathrm{t}_{1}}^{\mathrm{T}} \mathrm{I}(\mathrm{t}) \mathrm{dt}$

$\mathrm{SC}=\mathrm{C}_{1}\left[\frac{\mathrm{b}}{6}\left(3 \mathrm{t}_{1}{ }^{2} \mathrm{~T}-\mathrm{T}^{3}-2 \mathrm{t}_{1}{ }^{3}\right)-\frac{\mathrm{a}}{2}\left(\mathrm{~T}-\mathrm{t}_{1}\right)^{2}\right]$

Breaking cost: Total number of damageable/breakable units is

$$
\theta\left(Q_{0}\right)=\int_{0}^{t_{1}} B\left(Q_{0}\right) d t \quad=a_{1}\left(Q_{0}\right)^{r_{1}} t_{1}
$$

Where $\mathrm{B}\left(\mathrm{Q}_{0}\right)$ is breaking rate when inventory level is $\mathrm{Q}$ and it can be substituted as follows $\mathrm{B}\left(\mathrm{Q}_{0}\right)=\mathrm{a}_{1}\left(\mathrm{Q}_{0}\right)^{\mathrm{r}} \quad 0<r<1$.

As mentioned, selling price for each damaged item is $\mathrm{S}_{\mathrm{b}}=\mathrm{m}_{0} * \mathrm{P}, \quad 0<\mathrm{m}_{0}<1$ which is a multiple of last purchasing cost. So total selling price for damageable item is

$$
\begin{aligned}
& =\theta\left(Q_{0}\right)^{r} * m_{0} * P \\
& =a_{1}\left(Q_{0}\right)^{r} t_{1} * m_{0} * P
\end{aligned}
$$


M.Maragatham and G.Gnanvel

In this Case, annual interest earned isIE $=\mathrm{SI}_{\mathrm{e}}\left\{\int_{0}^{\mathrm{t}_{1}} \mathrm{I}(\mathrm{t}) \mathrm{dt}+\left(\mathrm{t}_{\mathrm{r}}-\mathrm{t}_{1}\right) \int_{\mathrm{t}_{1}}^{\mathrm{t}_{\mathrm{r}}} \mathrm{I}(\mathrm{t}) \mathrm{dt}\right\}$

$I E=S I_{e}\left\{\left[Q\left(t_{1}-\frac{\theta t_{1}{ }^{2}}{2}\right)-\frac{\theta t_{1}{ }^{2}}{2}\right]+\beta\left(t_{r}-t_{1}\right)\left[\frac{b}{6}\left(3 t_{1}{ }^{2} t_{r}-t_{r}{ }^{3}-2 t_{1}{ }^{3}\right)-\frac{a}{2}\left(t_{r}-t_{1}\right)^{2}\right]\right\}$

From the above arguments, the annual total relevant cost incurred at the retailer is

$\mathrm{TC}=\frac{1}{\mathrm{~T}}[\mathrm{OC}+\mathrm{PC}+\mathrm{HC}+\mathrm{SC}-\mathrm{IE}-$ Cost of D. items $]$

$\mathrm{TC}=\frac{1}{\mathrm{~T}}\left\{\mathrm{~A}+\mathrm{P} * \mathrm{Q}+\mathrm{h}\left[\mathrm{Q}\left(\mathrm{t}_{1}-\frac{\theta \mathrm{t}_{1}{ }^{2}}{2}\right)-\frac{\theta \mathrm{t}_{1}{ }^{2}}{2}\right]+\mathrm{C}_{1}\left[\frac{\mathrm{b}}{6}\left(3 \mathrm{t}_{1}{ }^{2} \mathrm{~T}-\mathrm{T}^{3}-2 \mathrm{t}_{1}{ }^{3}\right)-\right.\right.$

$\left.\frac{a}{2}\left(T-t_{1}\right)^{2}\right]-S_{e}\left\{\left[Q\left(t_{1}-\frac{\theta t_{1}{ }^{2}}{2}\right)-\frac{\theta t_{1}{ }^{2}}{2}\right]+\left(t_{r}-t_{1}\right)\left[\frac{b}{6}\left(3 t_{1}{ }^{2} t_{r}-t_{r}{ }^{3}-2 t_{1}{ }^{3}\right)-\right.\right.$

$\left.\left.\left.\frac{\mathrm{a}}{2}\left(\mathrm{t}_{\mathrm{r}}-\mathrm{t}_{1}\right)^{2}\right]\right\}-\mathrm{a}_{1}\left(\mathrm{Q}_{0}\right)^{\mathrm{r}} \mathrm{t}_{1} * \mathrm{~m}_{0} * \mathrm{P}\right\}$

For minimizing the total relevant cost per unit time, the approximate optimal values of $\mathrm{T}$ can be obtained by solving the following equation $\frac{\mathrm{dTC}}{\mathrm{dT}}=0$

$$
\begin{aligned}
\frac{\mathrm{dTC}}{\mathrm{dT}}=-\frac{1}{\mathrm{~T}^{2}}\{\mathrm{~A} & +\mathrm{P} * \mathrm{Q}+\mathrm{h}\left[\mathrm{Q}\left(\mathrm{t}_{1}-\frac{\theta \mathrm{t}_{1}{ }^{2}}{2}\right)-\frac{\theta \mathrm{t}_{1}{ }^{2}}{2}\right] \\
& -\mathrm{SI}_{\mathrm{e}}\left[\left(\mathrm{Q}\left(\mathrm{t}_{1}-\frac{\theta \mathrm{t}_{1}{ }^{2}}{2}\right)-\frac{\theta \mathrm{t}_{1}{ }^{2}}{2}\right)\right. \\
& \left.+\left(\mathrm{t}_{\mathrm{r}}-\mathrm{t}_{1}\right)\left(\frac{\mathrm{b}}{6}\left(3 \mathrm{t}_{1}{ }^{2} \mathrm{t}_{\mathrm{r}}-\mathrm{t}_{\mathrm{r}}{ }^{3}-2 \mathrm{t}_{1}{ }^{3}\right)-\frac{\mathrm{a}}{2}\left(\mathrm{t}_{\mathrm{r}}-\mathrm{t}_{1}\right)^{2}\right)\right]-\mathrm{a}_{1}\left(\mathrm{Q}_{0}\right)^{\mathrm{r}_{1}} \mathrm{t}_{1} * \mathrm{~m}_{0} \\
& \left.* \mathrm{P}-\mathrm{C}_{1}\left[\frac{\mathrm{bt}_{1}{ }^{3}}{3}-\frac{\mathrm{at}_{1}{ }^{2}}{2}\right]\right\}-\mathrm{C}_{1}\left[\frac{\mathrm{a}}{2}+\frac{\mathrm{bT}}{3}\right]=0
\end{aligned}
$$

This also satisfies the conditions

$$
\begin{aligned}
\frac{\mathrm{d}^{2} \mathrm{TC}}{\mathrm{dT}^{2}}=\frac{2}{\mathrm{~T}^{3}}\{\mathrm{~A} & +\mathrm{P} * \mathrm{Q}+\mathrm{h}\left[\mathrm{Q}\left(\mathrm{t}_{1}-\frac{\theta \mathrm{t}_{1}{ }^{2}}{2}\right)-\frac{\theta \mathrm{t}_{1}{ }^{2}}{2}\right] \\
& -\mathrm{SI}_{\mathrm{e}}\left[\left(\mathrm{Q}\left(\mathrm{t}_{1}-\frac{\theta \mathrm{t}_{1}{ }^{2}}{2}\right)-\frac{\theta \mathrm{t}_{1}{ }^{2}}{2}\right)\right. \\
& \left.+\left(\mathrm{t}_{\mathrm{r}}-\mathrm{t}_{1}\right)\left(\frac{\mathrm{b}}{6}\left(3 \mathrm{t}_{1}{ }^{2} \mathrm{t}_{\mathrm{r}}-\mathrm{t}_{\mathrm{r}}{ }^{3}-2 \mathrm{t}_{1}{ }^{3}\right)-\frac{\mathrm{a}}{2}\left(\mathrm{t}_{\mathrm{r}}-\mathrm{t}_{1}\right)^{2}\right)\right]-\mathrm{a}_{1}\left(\mathrm{Q}_{0}\right)^{\mathrm{r}_{1}} \mathrm{t}_{1} * \mathrm{~m}_{0} \\
& \left.* \mathrm{P}-\mathrm{C}_{1}\left[\frac{\mathrm{bt}_{1}{ }^{3}}{3}-\frac{\mathrm{at}_{1}{ }^{2}}{2}\right]\right\}-\mathrm{C}_{1}\left[\frac{\mathrm{b}}{3}\right]>0
\end{aligned}
$$

\subsection{Case III}

$$
\mathbf{t}_{\mathbf{1}}=\mathbf{t}_{\mathrm{r}}
$$

During the positive stock period $\left[0, \mathrm{t}_{1}\right]$. The inventory level decreases due to, both demand and damageable/breakable items will continue until the inventory level reaches zero at time. At the same time damageable items are replaced and permissible delay time $\left(t_{r}\right)$ is also equal to $t_{1}$. Again the inventory level decreases due to demand and damageable/breakableitems untilan inventory level become zero. Finally shortage is accumulated during $\left(t_{2}, T\right)$ which is backlogged when $t=T$. The inventory level at the time $t$ is governed by the following differential equations 
A Purchasing Inventory Model for Breakable items with Permissible Delay in Payments and Price Discount

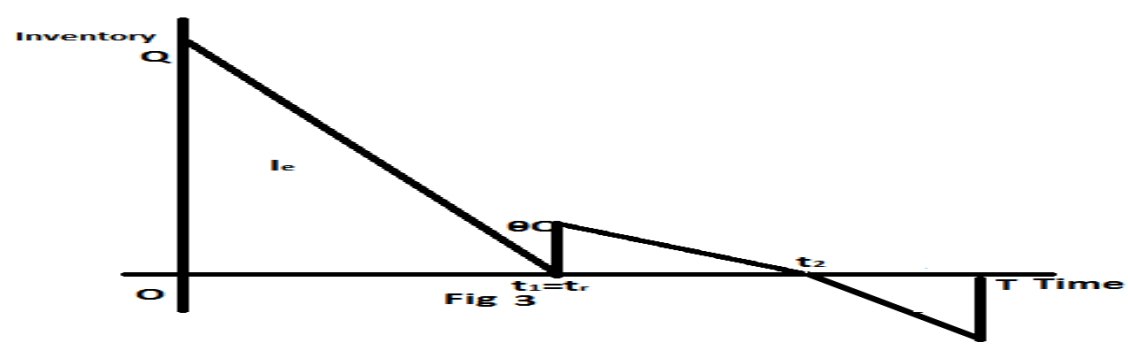

Figure 3:

From Equation $(1) \mathrm{I}(\mathrm{t})=\mathrm{Q}(1-\theta \mathrm{t})-$ at

$$
\frac{\mathrm{dI}(\mathrm{t})}{\mathrm{dt}}+\theta \mathrm{I}(\mathrm{t})=-\mathrm{D}(\mathrm{t}) \quad \mathrm{t}_{1}<t<\mathrm{t}_{2}
$$

With the boundary conditions $\quad I\left(t_{1}\right)=Q\left(1-\theta t_{1}\right)-a_{1}+\theta Q$

$I(t)=\left(Q\left(1-\theta t_{1}+\theta\right)-a t_{1}+\frac{b t_{1}}{\theta}\right)\left(\theta t_{1}-\theta t+1\right)+a\left(t_{r}-t\right)-\frac{b t_{r}}{\theta}$

With the boundary conditions $\quad \mathrm{I}\left(\mathrm{t}_{2}\right)=0$

$$
\begin{gathered}
\mathrm{t}_{2}=\frac{\left(\mathrm{Q}\left(1-\theta \mathrm{t}_{1}+\theta\right)-\mathrm{at}_{1}+\frac{\mathrm{bt}_{1}}{\theta}\right)\left(\theta \mathrm{t}_{1}+1\right)+\mathrm{at}_{1}-\frac{\mathrm{bt}_{1}}{\theta}}{\theta \mathrm{Q}\left(1-\theta \mathrm{t}_{1}+\theta\right)-\mathrm{a}\left(\theta \mathrm{t}_{1}-1\right)+\mathrm{bt}_{\mathrm{r}}} \\
\frac{\mathrm{dI}(\mathrm{t})}{\mathrm{dt}}=-\mathrm{D}(\mathrm{t}) \quad \mathrm{t}_{2}<t<T
\end{gathered}
$$

With the boundary conditions are $\quad I\left(t_{2}\right)=0, I(T)=-(1-\theta)\left[a+b\left(T-t_{2}\right)\right]$

$$
\mathrm{I}(\mathrm{t})=\left[\mathrm{a}(\mathrm{T}-\mathrm{t})+\frac{\mathrm{b}}{2}\left(\mathrm{~T}^{2}-\mathrm{t}^{2}\right)-(1-\theta)\left[\mathrm{a}+\mathrm{b}\left(\mathrm{T}-\mathrm{t}_{2}\right)\right]\right.
$$

The ordering cost is $\mathrm{OC}=\mathrm{A}$

The purchasing cost is $\mathrm{PC}=\mathrm{P}^{*} \mathrm{Q}+(1+\theta)\left[\mathrm{a}+\mathrm{b}\left(\mathrm{T}-\mathrm{t}_{2}\right)\right]$

The holding cost for the period $\left[0, \mathrm{t}_{2}\right]$

$$
\begin{aligned}
H C=h \int_{0}^{t_{2}} I(t) d t & H C=h\left[\int_{0}^{t_{1}} I(t) d t+\int_{t_{1}}^{t_{2}} I(t) d t\right] \\
H C=h\left[\left(Q t_{1}-\right.\right. & \left.\frac{t_{1}{ }^{2}}{2}(\theta Q+a)\right) \\
& +\left(Q\left(1-\theta t_{1}+\theta\right)-a_{1}+\frac{b t_{1}}{\theta}\right)\left(\frac{\theta\left(t_{2}-t_{1}\right)^{2}}{2}+\quad\left(t_{2}-t_{1}\right)\right) \\
& \left.+\frac{a\left(t_{2}-t_{1}\right)^{2}}{2}-\frac{b\left(t_{1} t_{2}-t_{1}{ }^{2}\right)}{\theta}\right]
\end{aligned}
$$

The shortage cost for the period $\left[t_{2}, T\right]$ is

$$
\begin{gathered}
S C=C_{1} \int_{t_{2}}^{T} I(t) d t \\
S C=C_{1}\left[\frac{b}{6}\left(2 T^{3}-3 T^{2} t_{2}+t_{2}{ }^{3}\right)+\left(\frac{a}{2}-b(1-\theta)\right)\left(T-t_{2}\right)^{2}-a(1-\theta)\left(T-t_{2}\right)\right]
\end{gathered}
$$




\section{M.Maragatham and G.Gnanvel}

During the permissible delay period when the account is not settled the retailer sells the goods and continues to accumulate sales revenue and earn the interest with rate $\mathrm{I}_{\mathrm{e}}$. Therefore the interest earned in the cycle period $\left[0, \mathrm{t}_{2}\right]$

$$
\begin{aligned}
& I E=S_{e} \int_{0}^{t_{2}} I(t) d t, \quad I E=S I_{e}\left[\int_{0}^{t_{1}} I(t) d t+\int_{t_{1}}^{t_{2}} I(t) d t\right] \\
& I E=S I_{e}\left\{\left(Q t_{1}-\frac{t_{1}{ }^{2}}{2}(\theta Q+a)\right)\right. \\
& +\left(\mathrm{Q}\left(1-\theta \mathrm{t}_{1}+\theta\right)-\mathrm{at}_{1}+\frac{\mathrm{bt_{1 }}}{\theta}\right)\left(\frac{\theta\left(\mathrm{t}_{2}-\mathrm{t}_{1}\right)^{2}}{2}+\left(\mathrm{t}_{2}-\mathrm{t}_{1}\right)\right) \\
& \left.+\frac{a\left(t_{2}-t_{1}\right)^{2}}{2}-\frac{b\left(t_{1} t_{2}-t_{1}^{2}\right)}{\theta}\right\}
\end{aligned}
$$

From the above arguments, the annual total relevant cost incurred at the retailer isTC $=$ $\frac{1}{\mathrm{~T}}[\mathrm{OC}+\mathrm{PC}+\mathrm{HC}+\mathrm{SC}-\mathrm{IE}] \mathrm{TC}=\frac{1}{\mathrm{~T}}\left\{\mathrm{~A}+\mathrm{P} * \mathrm{Q}+(1+\theta)\left[\mathrm{a}+\mathrm{b}\left(\mathrm{T}-\mathrm{t}_{2}\right)\right]+\right.$ $\mathrm{h}\left[\left(\mathrm{Qt}_{1}-\frac{\mathrm{t}_{1}{ }^{2}}{2}(\theta \mathrm{Q}+\mathrm{a})\right)+\left(\mathrm{Q}\left(1-\quad \theta \mathrm{t}_{1}+\theta\right)-\mathrm{at}_{1}+\frac{\mathrm{bt}_{1}}{\theta}\right)\left(\frac{\theta\left(\mathrm{t}_{2}-\mathrm{t}_{1}\right)^{2}}{2}+\right.\right.$ $\left.\left.\left(\mathrm{t}_{2}-\mathrm{t}_{1}\right)\right)+\frac{\mathrm{a}\left(\mathrm{t}_{2}-\mathrm{t}_{1}\right)^{2}}{2}-\frac{\mathrm{b}\left(\mathrm{t}_{1} \mathrm{t}_{2}-\mathrm{t}_{1}{ }^{2}\right)}{\theta}\right]+\quad \mathrm{C}_{1}\left[\frac{\mathrm{b}}{6}\left(2 \mathrm{~T}^{3}-3 \mathrm{~T}^{2} \mathrm{t}_{2}+\mathrm{t}_{2}{ }^{3}\right)+\left(\frac{\mathrm{a}}{2}-\right.\right.$ $\left.b(1-\theta))\left(T-t_{2}\right)^{2}-a(1-\theta)\left(T-t_{2}\right)\right]-\quad \operatorname{SI}_{e}\left[\left(\mathrm{Qt}_{1}-\frac{\mathrm{t}_{1}{ }^{2}}{2}(\theta \mathrm{Q}+\mathrm{a})\right)+\right.$ $\left.\left.\left(\mathrm{Q}\left(1-\theta \mathrm{t}_{1}+\theta\right)-\mathrm{at}_{1}+\frac{\mathrm{bt}}{\theta}\right)\left(\frac{\theta\left(\mathrm{t}_{2}-\mathrm{t}_{1}\right)^{2}}{2}+\quad\left(\mathrm{t}_{2}-\mathrm{t}_{1}\right)\right)+\frac{\mathrm{a}\left(\mathrm{t}_{2}-\mathrm{t}_{1}\right)^{2}}{2}-\frac{\mathrm{b}\left(\mathrm{t}_{1} \mathrm{t}_{2}-\mathrm{t}_{1}{ }^{2}\right)}{\theta}\right]\right\}$

Our objective is to minimize the total cost per unit time TC, The necessary condition for the total cost $\mathrm{TC}$ to be minimize is 
A Purchasing Inventory Model for Breakable items with Permissible Delay in Payments and Price Discount

$$
\begin{aligned}
\frac{\mathrm{dTC}}{\mathrm{dT}}=0 \quad \frac{\mathrm{dTC}}{\mathrm{dT}} & =-\frac{1}{\mathrm{~T}^{2}}\left\{\mathrm{~A}+\mathrm{P} * \mathrm{Q}+(1+\theta)\left[\mathrm{a}-\mathrm{bt}_{2}\right]\right. \\
& +\mathrm{h}\left[\left(\mathrm{Q} \mathrm{t}_{1}-\frac{\mathrm{t}_{1}{ }^{2}}{2}(\theta \mathrm{Q}+\mathrm{a})\right)\right. \\
& +\left(\mathrm{Q}\left(1-\theta \mathrm{t}_{1}+\theta\right)-\mathrm{at}_{1}+\frac{\mathrm{bt} \mathrm{t}_{1}}{\theta}\right)\left(\frac{\theta\left(\mathrm{t}_{2}-\mathrm{t}_{1}\right)^{2}}{2}+\left(\mathrm{t}_{2}-\mathrm{t}_{1}\right)\right) \\
& \left.+\frac{\mathrm{a}\left(\mathrm{t}_{2}-\mathrm{t}_{1}\right)^{2}}{2}-\frac{\mathrm{b}\left(\mathrm{t}_{1} \mathrm{t}_{2}-\mathrm{t}_{1}{ }^{2}\right)}{\theta}\right] \\
& +\mathrm{C}_{1}\left[\left(\frac{\mathrm{a}}{2}-\mathrm{b}(1-\theta)\right) \mathrm{t}_{2}{ }^{2}+\mathrm{a}(1-\theta) \mathrm{t}_{2}+\frac{\mathrm{b}}{6} \mathrm{t}_{1}{ }^{3}\right] \\
& -\mathrm{SI}_{\mathrm{e}}\left[\left(\mathrm{Qt} \mathrm{t}_{1}-\frac{\mathrm{t}_{1}{ }^{2}}{2}(\theta \mathrm{Q}+\mathrm{a})\right)\right. \\
& +\left(\mathrm{Q}\left(1-\theta \mathrm{t}_{1}+\theta\right)-\mathrm{at}_{1}+\frac{\mathrm{bt}}{\theta}\right)\left(\frac{\theta\left(\mathrm{t}_{2}-\mathrm{t}_{1}\right)^{2}}{2}+\left(\mathrm{t}_{2}-\mathrm{t}_{1}\right)\right) \\
& \left.\left.+\frac{\mathrm{a}\left(\mathrm{t}_{2}-\mathrm{t}_{1}\right)^{2}}{2}-\frac{\left.\mathrm{b}\left(\mathrm{t}_{1} \mathrm{t}_{2}-\mathrm{t}_{1}{ }^{2}\right)\right]}{\theta}\right]\right\}+\mathrm{C}_{1}\left(\frac{\mathrm{a}}{2}-\mathrm{b}\left(1-\theta-\frac{\mathrm{t}_{2}}{2}\right)\right)+\mathrm{C}_{1} 2 \mathrm{bT} \\
& =0
\end{aligned}
$$

This also satisfies the conditions

$$
\begin{aligned}
& \left(\mathrm{d}^{\wedge} 2 \mathrm{TC}\right) / \mathrm{dT}^{\wedge} 2>0\left(\mathrm{~d}^{\wedge} 2 \mathrm{TC}\right) / \mathrm{dT}^{\wedge} 2 \\
& =2 / \mathrm{T}^{\wedge} 3\left\{\boldsymbol { \square } \left(\mathrm{~A}+\mathrm{P} * \mathrm{Q}+(1+\theta)\left[\mathrm{a}-\mathrm{bt} \_2\right]+@ \mathrm{~h}\left[\left(\mathrm{Qt} \_1\right.\right.\right.\right. \\
& \text { - [t_1 } \left.1 \wedge^{\wedge} 2 / 2(\theta \mathrm{Q}+\mathrm{a})\right)+\left(\mathrm{Q}\left(1-\theta \mathrm{t}_{-} 1+\theta\right)-\mathrm{at} \_1\right. \\
& \left.+\mathrm{bt}_{-} 1 / \theta\right)\left(\left(\theta\left(\mathrm{t} \_2-\mathrm{t} \_1\right)^{\wedge} 2\right) / 2+\left(\mathrm{t} \_2-\mathrm{t} \_1\right)\right)+\left(\mathrm{a}\left(\mathrm{t} \_2-\mathrm{t} \_1\right)^{\wedge} 2\right) / 2 \\
& -\mathrm{b}\left(\mathrm{t} \_1 \mathrm{t} \_2-\left[\mathrm{t} \_1 \rrbracket \wedge 2\right) / \theta\right] \\
& +\mathrm{C}_{-} 1\left[(\mathrm{a} / 2-\mathrm{b}(1-\theta)) \text { [t_2 } 2 \wedge 2+\mathrm{a}(1-\theta) \mathrm{t} \_2+\mathrm{b} / 6 \text { [t_1 } \wedge^{\wedge} 3\right]-\text { SI_e [(Qt_1 } \\
& \text { - 『t_1 } \left.1 \wedge^{\wedge} 2 / 2(\theta \mathrm{Q}+\mathrm{a})\right)+\left(\mathrm{Q}\left(1-\theta \mathrm{t} \_1+\theta\right)-\right.\text { at_1 } \\
& \left.+b_{-} 1 / \theta\right)\left(\left(\theta\left(\mathrm{t} \_2-\mathrm{t} \_1\right)^{\wedge} 2\right) / 2+\left(\mathrm{t} \_2-\mathrm{t} \_1\right)\right)+\left(\mathrm{a}\left(\mathrm{t} \_2-\mathrm{t} \_1\right)^{\wedge} 2\right) / 2 \\
& \left.\left.-\mathrm{b}\left(\mathrm{t} \_1 \mathrm{t} \_2-\left[\mathrm{t} \_1 \rrbracket \wedge 2\right) / \theta\right]\right)\right\} \llbracket+\mathrm{C} \rrbracket \_12 \mathrm{~b}>0
\end{aligned}
$$

\section{Numerical example}

Consider an inventory system with the following data

\begin{tabular}{|l|l|l|l|l|l|l|l|l|l|l|l|l|l|l|}
\hline S.No & 1 & 2 & 3 & 4 & 5 & 6 & 7 & 8 & 9 & 10 & 11 & 12 & 13 & 14 \\
\hline Parameters & $\mathrm{A}$ & $\mathrm{a}$ & $\mathrm{b}$ & $\mathrm{I}_{\mathrm{P}}$ & $\mathrm{I}_{\mathrm{e}}$ & $\mathrm{h}$ & $\mathrm{P}$ & $\theta$ & $\mathrm{Q}$ & $\mathrm{t}_{\mathrm{r}}$ & $\mathrm{c}_{1}$ & $\mathrm{~S}$ & $\mathrm{r}$ & $\mathrm{m}_{0}$ \\
\hline Values & 150 & 230 & 0.6 & 0.10 & 0.12 & 0.10 & 15 & 0.15 & 65 & 0.30 & 4 & 17 & 0.02 & 0.15 \\
\hline
\end{tabular}

For the above parametric values $t_{1}=0.27$, here $t_{r}=0.30>t_{1}$. So using case: 2 , optimal value of $\mathrm{T}^{*}=1(1.0379)$ and $\mathrm{TC}^{*}=563$ in appropriate units.

\section{Conclusion}

In this paper, an inventory model is developed in which the optimal cycle time is determined to minimize the total inventory cost. The shortages are allowed and are 


\section{M.Maragatham and G.Gnanvel}

completely backlogged. The proposed model is discussed in three cases. Finally, numerical example is given to illustrate the model.

\section{REFERENCES}

1. S.K Goyal, Economic order quantity under conditions of permissible delay in payments, J Operation Research Scientific Society 36 (1985) 335-338.

2. J.Ray, A non linear EOQ model with the effect of trade credit, International Journal of Non Linear Science, 17 (2014) 135-144.

3. M.Sajadifar and A.Mavaji, An inventory model with demand dependent replenishment rate for damageable item and shortage, International Conference on Industrial Engineering and Operations Management Bali, Indonesia, January 7-9, 2014.

4. M.Kumar, A.Chauhan and R.Kumar, A deterministic inventory model for deteriorating items with price dependent demand and time varying holding cost under trade credit, International Journals of Soft Computing and Engineering, 2 (2012).

5. M.Palanivel and R.Uthayakumar, An EOQ model for non instantaneous deteriorating items with power demand, time dependent holding cost, partial backlogging and permissible delay in payments, International Journal of Mathematical Computational Sciences, 8(8) 2014.

6. A.Thangam, Retailers inventory system in atwo level trade credit finanacing with selling price discount and partial order cancellations, Journals of Industrial Engineering International, 11(2) (2015) 159-170. 